\title{
Meteor Showers of the Earth-crossing Asteroids
}

\section{Babadzhanov Pulat and Kokhirova Gulchekhra}

Institute of Astrophysics, Ac. of Sci. of Tajikistan

email: kokhirova2004@mail.ru

\begin{abstract}
The results of search for meteor showers associated with the asteroids crossing the Earthfs orbit and moving on comet-like orbits are given. It was shown that among 2872 asteroids discovered till 1.01.2005 and belonging to the Apollo and Amor groups, 130 asteroids have associated meteor showers and, therefore, are the extinct cometary nuclei.
\end{abstract}

